\title{
Projected Iterative MVDR Beamforming for Null Broadening and First Sidelobe Suppression in the Presence of DOA Mismatch
}

\begin{abstract}
Raungrong Suleesathira ${ }^{+}$
Department of Electronic and Telecommunication Engineering, Faculty of Engineering

King Mongkut's University of Technology Thonburi, Thung-khru, Bangmod, Bangkok, 10140 Thailand

Abstract. Although the minimum variance distortionless response (MVDR) beamformer can keep the desired signal from interfering signals, however, it has notch null and no sidelobe level control which may lead to performance degradation in the case of scattering environment or unexpected interfering signals such as rapidly moving jammer environment. It also has high sidelobe levels for either low number of samples or high input signal to noise ratio (SNR). The projected iterative MVDR (PI-MVDR) beamformer is presented to broaden the width of nulls, suppress the first sidelobe level and support the case of low number of samples no matter of low or high input SNR. The averaged covariance matrix used in the PI-MVDR beamformer can overcome the circumstance of low number of samples over any input SNR value. Projecting the averaged covariance matrix on to the interference space enables the iterative MVDR beamformer to steer a mainbeam toward the desired signal direction and make a broad null in the direction of an unwanted signal. To reduce the first sidelobe level, find the direction corresponding to the strongest sidelobe close to the mainbeam which is considered as a direction of arrival (DOA) of a hypothetical interference signal and employ the PIMVDR beamformer to produce extra wide null. The steering vector of the desired signal is estimated to illustrate the robustness of the PI-MVDR beamformer in the presence of DOA mismatch. Simulation results demonstrated the achievement of the presented approach.
\end{abstract}

Keywords: beamformer, covariance matrix, direction of arrival

\section{Introduction}

Array beamforming methods make use of the spatial dimension to combat interference signals and noise by producing nulls at the direction of interference signals and a mainbeam at the direction of the desired signal. A variety of issues have been challenged to be solved. The performance can be severely degraded if the DOA is spread due to multipath propagation or rapidly moving interferences. Forming broad nulls around the directions of interferences can solve the problem since the broaden null can reject the interfering sources not only from a specific direction but from a specific spatial region as well. As a result, null broadening allows the interferer to move in a certain area. New interferences might exist during the time span processing. Suppressing the sidelobes especially the ones close to the mainbeam would be benefit to reject the new interferences disturbing the desired signal. Most beamformers dramatically degraded when received signal is either taken over few samples or strong, the weights might not only give a proper beampattern corresponding to the DOA of the received signal and high sidelobe as well. Another challenge is the sensitiveness to the mismatch between the actual and presumed steering vector of the desired signal.

Covariance matrix taper (CMT) is a classical approach of null broadening [1]. Adaptive variable diagonal loading combined with the CMT approach is presented in [2] for null broadening beamforming to develop the CMT. The CMT and projection are combined, the null depth is deeper than that of the CMT approach [3]. The researches that focus only on the sidelobe reduction have been conducted. Recently, an iterative beamforming is proposed in order to improve the three conventional beamformers by placing extra

\footnotetext{
${ }^{+}$Corresponding author. Tel.: +6695257197
}

E-mail address: raungrong.sul@kmutt.ac.th 
nulls to achieve the desired sidelobe level [4]. Efforts to broaden nulls and control the sidelobe levels have been done. In [5], the CMT is constructed to broaden the width of nulls for interference signal sources. Constraint of nulls and sidelobe levels are used to guarantee that the level is strictly lower than the prescribed threshold value. Certainly, the optimal solution needs complex computation. In [6], it uses the CMT to broaden the width of nulls and uses the support vector match regression to control the sidelobe level.

The rest of paper is organized as follows. The signal model is described in Section 2. The conventional MVDR beamforming is updated in Section 3. The averaged covariance matrix is given in Section 4. Section 5 presents an estimation of the steering vector of the desired signal used in the presence of DOA mismatch. The projected iterative MVDR beamformer is proposed in Section 6 for null broadening and first sidelobe suppression. The evaluation of the proposed method is verified in the simulation results of Section 7. Finally, conclusions are drawn in Section 8.

\section{Signal Model}

Consider a uniform linear array (ULA) comprising $L$ omni-directional antenna elements with equispaced $d$ as shown in Fig. 1. A narrowband source of interest and interferences are transmitted in the far field region of the array. The received signal at the sampling time $k$ is mathematically represented as [7]

$$
\mathbf{x}(k)=s_{d}(k) \mathbf{a}\left(\theta_{d}\right)+\sum_{i=1}^{I} s_{i}(k) \mathbf{a}\left(\theta_{i}\right)+\mathbf{n}(k)
$$

The $L \times 1$ received signal vector $\mathbf{x}(k)=\left[\begin{array}{llll}x_{1}(k) & x_{2}(k) & \ldots & x_{L}(k)\end{array}\right]^{T}$ consists of the desired signal, a sum of $I$ interference signals $((I+1)<L)$ and an additive noise. Let denote $s_{d}(k)$ be the desired signal waveform in the directions of arrival (DOA) of $\theta_{d}, s_{i}(k)$ be the $i$ th interference waveform in the DOA of

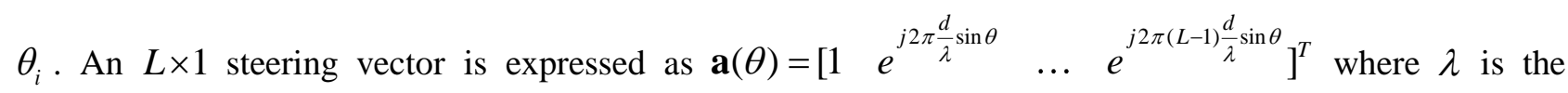
signal wavelength, $(\cdot)^{T}$ denotes the transpose operation. The steering matrix with columns of the steering vector is given as $\mathbf{A}=\left[\begin{array}{llll}\mathbf{a}\left(\theta_{d}\right) & \mathbf{a}\left(\theta_{1}\right) & \ldots & \mathbf{a}\left(\theta_{I}\right)\end{array}\right]$. Then, the received signal in Eq. (1) can be rewritten as

$$
\mathbf{x}(k)=\mathbf{A s}(k)+\mathbf{n}(k)
$$

where $\mathbf{s}(k)=\left[\begin{array}{llll}s_{d}(k) & s_{1}(k) & \ldots & s_{I}(k)\end{array}\right]^{T}$ is an $(I+1) \times 1$ vector of the desired signal and interferences impinging on the antenna array. Assume that the desired and interference signals are uncorrelated, i.e.,

$$
E\left[s_{d}(k) s_{d}^{*}(k)\right]=p_{d}, E\left[s_{d}(k) s_{i}^{*}(k)\right]=0 \text { for } i=1, \ldots I \text { and } E\left[s_{i}(k) s_{j}^{*}(k)\right]= \begin{cases}0 & i \neq j \\ p_{i} & i=j\end{cases}
$$

where $p_{d}$ and $p_{i}$ denotes the power of the desired signal and the $i$ th interference signal, respectively. $\mathbf{n}(k)=\left[\begin{array}{lllll}n_{1}(k) & n_{2}(k) & \ldots & n_{L}(k)\end{array}\right]^{T}$ is an $L \times 1$ complex noise vector with zero mean and variance $\sigma_{n}^{2}$. In fact, the directional sources and the noise are uncorrelated, i.e., $E\left[s_{d}(k) n_{l}^{*}(k)\right]=0$ and $E\left[s_{i}(k) n_{l}^{*}(k)\right]=0$ for $i=1, \ldots, I$ and $l=1, \ldots, L$. The noise on different elements is also assumed to be uncorrelated and independent, i.e. $E\left[n_{j}(k) n_{l}^{*}(k)\right]=E\left[n_{j}(k)\right] E\left[n_{l}^{*}(k)\right]=\left\{\begin{array}{cc}0 & j \neq l \\ \sigma_{n}^{2} & j=l\end{array}\right.$ where $E[\bullet]$ denotes the expectation operator and $(\cdot)^{*}$ denotes the complex conjugate.

The covariance matrix of the received signal is formulated as $\mathbf{R}_{x}=E\left[\mathbf{x}(k) \mathbf{x}^{H}(k)\right]$. By using $\mathbf{x}(k)$ in Eq. (1), it becomes

$$
\mathbf{R}_{x}=p_{d} \mathbf{a}\left(\theta_{d}\right) \mathbf{a}^{H}\left(\theta_{d}\right)+\sum_{i=1}^{I} p_{i} \mathbf{a}\left(\theta_{i}\right) \mathbf{a}^{H}\left(\theta_{i}\right)+\sigma_{n}^{2} \mathbf{I}_{L}
$$


where $\mathbf{I}_{L}$ is the $L \times L$ identity matrix and $(\cdot)^{H}$ denotes the complex conjugate transposition operation. Eq. (3) can be considered as a sum of the covariance matrix of the desired signal as $\mathbf{R}_{d}=p_{d} \mathbf{a}\left(\theta_{d}\right) \mathbf{a}^{H}\left(\theta_{d}\right)$ and the interference-plus-noise covariance matrix as $\mathbf{R}_{i+n}=\sum_{i=1}^{I} p_{i} \mathbf{a}\left(\theta_{i}\right) \mathbf{a}^{H}\left(\theta_{i}\right)+\sigma_{n}^{2} \mathbf{I}_{L}$.

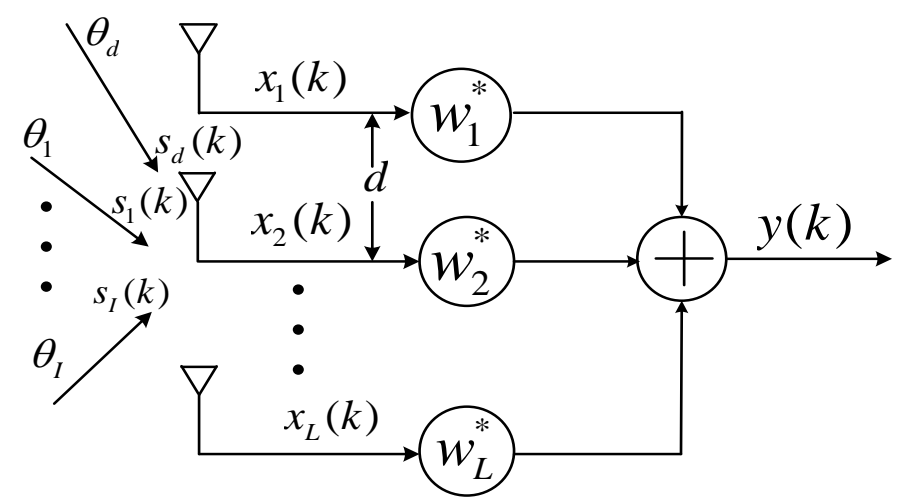

Fig. 1: A ULA of $L$ antenna elements receiving $(I+1)$ directional sources

\section{Iterative MVDR Beamformer}

The output signal can be calculated by $y(k)=\mathbf{w}^{H} \mathbf{x}(k)$ where $\mathbf{w}=\left[\begin{array}{llll}w_{1} & w_{2} & \ldots & w_{L}\end{array}\right]^{T}$ is an $L \times 1$ weight vector. Given $\mathbf{w}$, the output SINR is found by

$$
\operatorname{SINR}(\mathbf{w})=\frac{p_{d}\left|\mathbf{w}^{H} \mathbf{a}\left(\theta_{d}\right)\right|^{2}}{\mathbf{w}^{H} \mathbf{R}_{i+n} \mathbf{w}}
$$

Under assumption that the steering vector of the desired signal $\mathbf{a}\left(\theta_{d}\right)$ is known precisely, the minimum variance distortionless response (MVDR) beamformer is obtained by minimizing the denominator of Eq. (4), i.e., minimizing the variance/power of interference and noise while keeping the numerator of Eq. (4) fixed, i.e., ensuring the output desired signal without distortion towards the direction of the desired source. This is equivalent to a linearly constrained quadratic optimization problem as

$$
\min _{\mathbf{w}} \mathbf{w}^{H} \mathbf{R}_{i+n} \mathbf{w} \quad \text { subject to } \mathbf{w}^{H} \mathbf{a}\left(\theta_{d}\right)=1 .
$$

Using the Lagrange multiplier, the optimal solution for this weight vector is obtained as [8]

$$
\mathbf{w}_{M D V R}=\alpha \mathbf{R}_{i+n}^{-1} \mathbf{a}\left(\theta_{d}\right)
$$

where $\alpha=1 / \mathbf{a}^{H}\left(\theta_{d}\right) \mathbf{R}_{i+n}^{-1} \mathbf{a}\left(\theta_{d}\right)$ and $(\cdot)^{-1}$ denotes the matrix inversion. To avoid the inverse matrix calculation in Eq. (6), the solution can be obtained by weight adjustment as [8]

$$
\mathbf{w}_{m+1}=\mathbf{w}_{m}-c\left(\mathbf{R}_{i+n} \mathbf{w}_{m}-\mathbf{a}\left(\theta_{d}\right)\right) .
$$

For fast convergence, set the constant $c=2 /\left(\lambda_{\min }+\lambda_{\max }\right)$ where $\lambda_{\min }$ and $\lambda_{\max }$ are the minimum and maximum eigenvalues of $\mathbf{R}_{i+n}$, respectively. For some amount of number of iterations, the weight vector in Eq. (7) converges to $\mathbf{w}_{M D V R}[9-10]$.

\section{Averaged Covariance Matrix}

Since it is difficult to obtain $\mathbf{R}_{i+n}$ in practice, this matrix is commonly replaced by the sample covariance matrix of the received signal with limited number of snapshots $K$ as $\hat{\mathbf{R}}_{x}=\frac{1}{K} \sum_{k=0}^{K-1} \mathbf{x}(k) \mathbf{x}^{H}(k)$.

When $K \rightarrow \infty, \hat{\mathbf{R}}_{x}$ will converge to the theoretical covariance matrix $\mathbf{R}_{x}$. There are two drawbacks of replacement. First, when $K$ is small, a gap between $\hat{\mathbf{R}}_{x}$ and $\mathbf{R}_{x}$ becomes larger. The limited number of snapshots can cause the disturbance of small eigenvalues corresponding to the noise subspace. As a result, the performance of the beamforming will be degraded due to the rise of sidelobes. Secondly, the 
performance degradation will be significant as input SNR increases which can be proven by substituting $\mathbf{R}_{x}=\mathbf{R}_{d}+\mathbf{R}_{i+n}$ into $\mathbf{w}^{H} \mathbf{R}_{x} \mathbf{w}$. Since $\mathbf{w}^{H} \mathbf{a}\left(\theta_{d}\right)=1$, it yields as

$$
\mathbf{w}^{H} \mathbf{R}_{x} \mathbf{w}=p_{d}+\mathbf{w}^{H} \mathbf{R}_{i+n} \mathbf{w} .
$$

Thus, as the power $p_{d}$ increases, such replacement can cause large error.

Consider the case of low number of samples $K=30$ and high input SNR $\left(10 \log _{10}\left(p_{d} / \sigma_{n}^{2}\right)\right) 3 \mathrm{~dB}$. Fig. 2 shows the comparison the optimal beamformer $\mathbf{w}_{M D V R}$ using the sample covariance matrix of the receive signal $\hat{\mathbf{R}}_{x}$ and the sample covariance matrix of the interference-plus-noise signal given by $\hat{\mathbf{R}}_{i+n}=\frac{1}{K} \sum_{k=0}^{K-1}\left(\mathbf{x}_{i}(k)+\mathbf{n}(k)\right)\left(\mathbf{x}_{i}(k)+\mathbf{n}(k)\right)^{H}$. A ULA of 10 elements is used with inter-element spacing $d=\lambda / 2$. The desired signal is taken at $\theta_{d}=0^{\circ}$ and two interferences are taken at $\theta_{1}=-45^{\circ}$ and $\theta_{2}=45^{\circ}$. The beampattern using $\hat{\mathbf{R}}_{x}$ (blue line) gives the bad mainbeam and shallow nulls. For the beampattern using $\hat{\mathbf{R}}_{i+n}$ (red line), the mainbeam is unity at $\theta_{d}=0^{\circ}$ and the two notch nulls are at $\theta_{1}=-45^{\circ}$, $\theta_{2}=45^{\circ}$. The result assures that the replacement $\mathbf{R}_{i+n}$ by $\hat{\mathbf{R}}_{x}$ degrades the performance of the MVDR beamformer if high input SNR is present in the sample data or low number of snapshot is available.

Then, an estimate of the interference-plus-noise covariance matrix is needed to achieve a better beampattern. The spatial spectrum distribution in all direction is first created as

$$
P(\theta)=\frac{1}{\mathbf{a}^{H}(\theta) \mathbf{E}_{i+n} \mathbf{E}_{i+n}^{H} \mathbf{a}(\theta)}
$$

where $\mathbf{E}_{i+n}$ is the interference plus noise subspace found by applying the singular value decomposition to $\hat{\mathbf{R}}_{x}$ as $\hat{\mathbf{R}}_{x}=\sum_{l=1}^{L} e_{l} \mathbf{e}_{l} \mathbf{e}_{l}^{H}$. Descend the order of eigenvalues $e_{l}$ as $e_{1}>e_{2}, \ldots,>e_{L}$. According to Eqs. (1) and (2), the received signal is the addition of source of interest, interferences and noise. Consequently, the interference plus noise subspace consists of the eigenvectors belong to the small eigenvalues built as $\mathbf{E}_{i+n}=\left[\begin{array}{llll}\mathbf{e}_{2} & \mathbf{e}_{3} & \ldots & \mathbf{e}_{L}\end{array}\right]$. The interference-plus-noise covariance matrix can be reconstructed by utilizing the spectrum to integrate over a region separated from the desired directions [11].

$$
\tilde{\mathbf{R}}_{i+n}=\int_{\bar{\Theta}} P(\theta) \mathbf{a}(\theta) \mathbf{a}^{H}(\theta) d \theta
$$

where $\bar{\Theta}$ is the complement sector of $\Theta$ and $\Theta$ is an angular sector where the desired signal is located. $\Theta \cup \bar{\Theta}$ covers the whole spatial domain while $\Theta \cap \bar{\Theta}$ is empty.

However, at low input SNR, the replacement of $\mathbf{R}_{i+n}$ by $\hat{\mathbf{R}}_{x}$ results in a small error, since the desired signal power $p_{d}$ is small. At high input SNR, we need an estimate instead of the replacement. In order to select the proper covariance matrix corresponding to the input SNR value, $\mathbf{R}_{i+n}$ will be replaced by the averaged covariance matrix $\mathbf{R}_{\text {ave }}$ as [12]

$$
\mathbf{R}_{a v e}=\alpha \tilde{\mathbf{R}}_{i+n} /\left\|\tilde{\mathbf{R}}_{i+n}\right\|_{F}+(1-\alpha) \hat{\mathbf{R}}_{x} /\left\|\hat{\mathbf{R}}_{x}\right\|_{F}
$$

where $\|\cdot\|_{F}$ is the frobenius norm. The parameter $\alpha$ is defined as

$$
\alpha=\frac{\mathbf{a}^{H}\left(\theta_{d}\right) \hat{\mathbf{R}}_{x} \mathbf{a}\left(\theta_{d}\right)}{\left\|\hat{\mathbf{R}}_{x}\right\|_{F}\left\|\mathbf{a}\left(\theta_{d}\right)\right\|^{2}}
$$

where $\|\cdot\|$ is the norm of a vector. The value of $\alpha$ is between zero and one which can be used as an indicator of the desired signal power compared with the interference signal power. When $\alpha$ is equal to zero, it means that there is no signal from the direction $\theta_{d}$, then $\hat{\mathbf{R}}_{x}$ can be used for low input SNR. When $\alpha$ is equal to one, it means that the eigenvector associated to the largest eigenvalue of $\hat{\mathbf{R}}_{x}$ is equal to $\mathbf{a}\left(\theta_{d}\right)$, then $\tilde{\mathbf{R}}_{i+n}$ should be used for high input SNR. Figure 3 shows the $\alpha$ values increase versus the input SNR. 


\section{Steering Vector of the Desired Signal Estimation}

When the DOA of the desired signal mismatch exists, the performance of the MVDR beamformer and the iterative MVDR beamformer will be significantly degraded. Also, the value of $\alpha$ will reflect incorrect meanings. The mismatch between the actual DOA $\theta_{d}$ and the presumed DOA $\bar{\theta}_{d}$ falls in the uncertainty region defined as $\Theta=\left[\theta_{d}-\Delta_{d}, \theta_{d}+\Delta_{d}\right]$ where $\Delta_{d}$ is the angular sector of mismatch. Since only one desired signal assumed in the uncertainty region, the eigenvector with the largest eigenvalue of the constructed matrix will be the steering vector estimate. Build a matrix over the uncertainty region as $\mathbf{V}=\int_{\Theta} \frac{\mathbf{a}(\theta) \mathbf{a}^{H}(\theta)}{\mathbf{a}(\theta) \hat{\mathbf{R}}_{x}^{-1} \mathbf{a}^{H}(\theta)}$. Apply the singular value composition to the matrix $\mathbf{V}$ to obtain the most principle eigenvector $\mathbf{v}_{d s}$. The steering vector of the desired signal can be estimated as $\overline{\mathbf{a}}\left(\theta_{d}\right)=\frac{\mathbf{v}_{d s}}{\left|v_{d s}\right|}$ where $\left|v_{d s}\right|$ is the absolute value of the first element in the vector $\mathbf{v}_{d s}[13]$.

\section{Projected Iterative MVDR Beamformer}

For low input SNR, the weight vector $\mathbf{w}_{M V D R}$ is able to form a mainbeam in the look direction of the desired signal and nulls in the undesired directions belong to the DOAs of interference signals. However, the null is notch and it does not take into account the sidelobe levels. Since a moving interference is a serious problem in the antenna array, we then assume that the direction of the desired signal $\theta_{d}$ is constant whereas $\theta_{i}$, on the other hand, is not constant. In a local time-varying scattering environment, the interference direction is randomly selected from the interval $\left(\theta_{i}-\Delta \theta / 2, \theta_{i}-\Delta \theta / 2\right)$ where $\Delta \theta$ is the total direction change so-called the angular spread which is used to determines the null width.

New interference signals may arise during the time span that a certain data sample set is processed. Since this sample set does not contain these new interference signals, then no null is create to cancel, thus resulting in SINR degradation. Especially, if these new interference signals have DOA close to the directions of the mainlobe which is the DOA of the desired signal. In such case, a low first sidelobe beside the mainlobe would help to keep the SINR at high levels. The nulls can be broadened and the sidelobe level can be controlled by the following projection transform [3].

The projection approach begins with constructing the correlation matrix of $I$ interference signals as $\mathbf{Z}_{P}=\sum_{i=1}^{I} \mathbf{a}\left(\theta_{i}\right) \mathbf{a}^{H}\left(\theta_{i}\right)$. Next, the matrix $\mathbf{Z}_{p}$ is tapered by $\mathbf{R}_{P}=\mathbf{Z}_{P} \circ \mathbf{T}$ where $\circ$ represents Hadamard product and the sample of the $a$ th row and $b$ th column of the tapered matrix $\mathbf{T}$ is expressed as $T_{a b}=\frac{\sin ((a-b) \Delta \theta)}{(a-b) \Delta \theta}$. Decompose the matrix $\mathbf{R}_{P}$ as $\mathbf{R}_{P}=\sum_{l=1}^{L} v_{l} \mathbf{v}_{l} \mathbf{v}_{l}^{H}$. By chosing $J$ eigenvectors belonging to the $J$ largest eigenvalues, the projection matrix is $\mathbf{T}_{P}=\sum_{l=1}^{J<L} \mathbf{v}_{l} \mathbf{v}_{l}^{H}$. Then, the projected covariance matrix can be modified as $\mathbf{R}_{P R O}=\mathbf{T}_{P} \mathbf{R}_{\text {ave }} \mathbf{T}_{P}^{H}$. The projected iterative MVDR (PI-MVDR) beamformer is obtained by replacing $\mathbf{R}_{i+n}$ with $\mathbf{R}_{P R O}$ as [9-10]

$$
\mathbf{w}_{m+1}=\mathbf{w}_{m}-c\left(\mathbf{R}_{P R O} \mathbf{w}_{m}-\mathbf{a}\left(\theta_{d}\right)\right) .
$$

Let $\mathbf{w}_{P I-M V D R}$ denotes the weight vector after the iteration is terminated.

After obtaining $\mathbf{w}_{P I-M V D R}$, the next step is to suppress the sidelobe levels. The proposed algorithm to suppress the first sidelobe levels is summarized by the flowchart drawn in Fig. 4.

\section{Simulation Results}

A 30-element ULA with a spacing of half wavelength is used. The additive noise is modeled as a complex Guassian spatially and temporally white process with zero mean. Two interfering sources 
$(I=2)$ are from directions of $\theta_{1}=-45^{\circ}$ and $\theta_{2}=45^{\circ}$ with the angular spread $\Delta \theta=10^{\circ}$. The input SNR is equal to $3 \mathrm{~dB}$ and the INR (interference to noise ratio) is equal to $6 \mathrm{~dB}$. The number of snapshot is set to $K=30$. The actual DOA of the desired signal is $\theta_{d}=5^{\circ}$. The angular sector of mismatch is $\Delta_{d}=2^{\circ}$. The possible angular sector of the desired signal is located $\Theta=\left[3^{\circ} 7^{\circ}\right]$, so the complement sector is $\bar{\Theta}=\left[-90^{\circ} 3^{\circ}\right) \cup\left(7^{\circ} 90^{\circ}\right]$. The number of weight adjustment to terminate the iteration is 15 .

Figure 5 shows the PI-MVDR beamformer by using $\hat{\mathbf{R}}_{x}, \hat{\mathbf{R}}_{i+n}, \tilde{\mathbf{R}}_{i+n}$ and $\mathbf{R}_{\text {ave }}$ to generate $\mathbf{R}_{P R O}$. The beam patterns using $\hat{\mathbf{R}}_{x}, \hat{\mathbf{R}}_{i+n}$ (blue and purple lines) is bad when the number of snapshot is few and the input SNR is high. The beampattern using $\tilde{\mathbf{R}}_{i+n}$ (black line) can reduce the sidelobe levels but still has shallow nulls since the interference and noise subspace generated is not good for low number of samples. The beampattern using $\mathbf{R}_{\text {ave }}$ (red line) provides not only the right mainbeam at the desired direction $\theta_{d}=5^{\circ}$ and also broad nulls between $-50^{\circ}$ to $-40^{\circ}$ and $40^{\circ}$ to $50^{\circ}$ corresponding to the angular spread of each interference direction.

In Figure 6, the beampattern after suppression (red line) can reduce the first sidelobe levels as marked by the two ellipses and still keep the null width and the mainbeam unchanged. Also, this does not affect the position of the nulls initially placed to cancel the interference signals.

It can be observed in Figure 7 that the output SINR increases significantly especially in the negative range input SINR. Due to the high gain of the output SINR over the input SINR, it implies that the interference signals at $\theta_{1}=-45^{\circ}, \theta_{2}=45^{\circ}$ are mitigated efficiently.

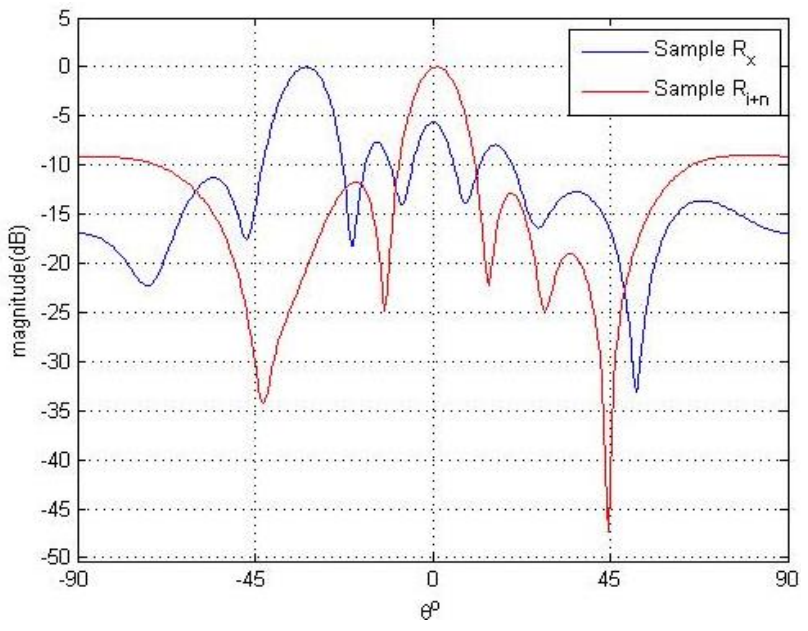

Fig. 2: Beampatterns by the MVDR Beamformer using $\hat{\mathbf{R}}_{x}$ and $\hat{\mathbf{R}}_{i+n}$

Projected iterative MVDR Beamforming to calculate $\mathbf{W}_{P I-M V D R}$

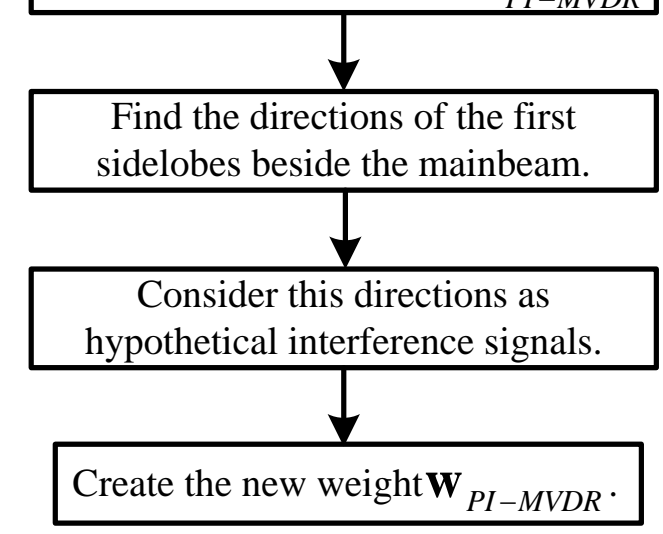

Fig. 4: Flowchart of the sidelobe suppression

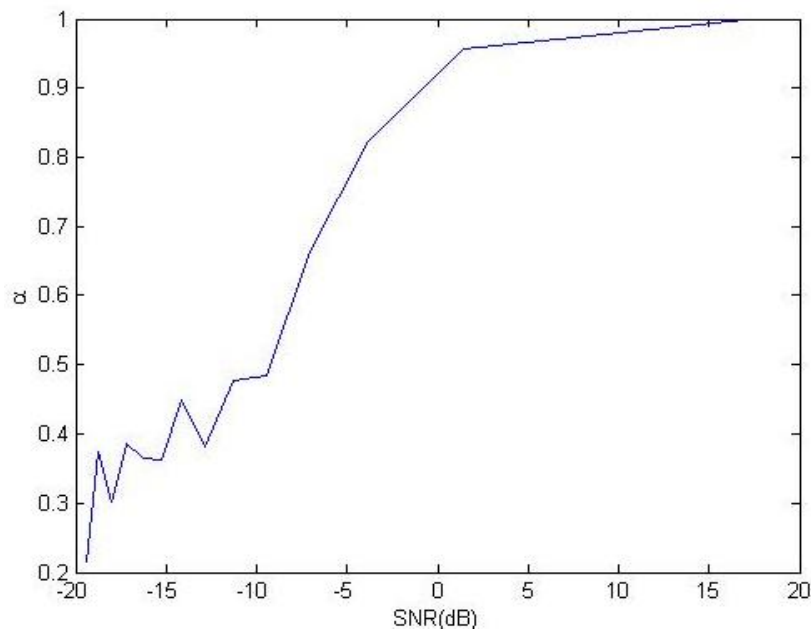

Fig. 3: The values of $\alpha$ versus the input SNR

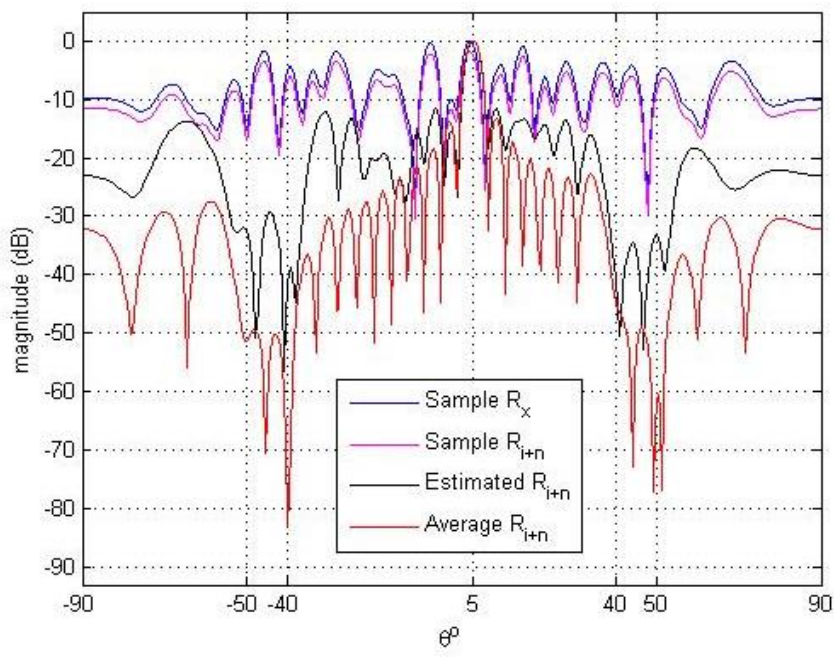

Fig. 5: Beampattrns by the PI-MVDR beamformer using $\hat{\mathbf{R}}_{x}, \hat{\mathbf{R}}_{i+n}, \quad \tilde{\mathbf{R}}_{i+n}$ and $\mathbf{R}_{a v e}$ to generate $\mathbf{R}_{P R O}$ 


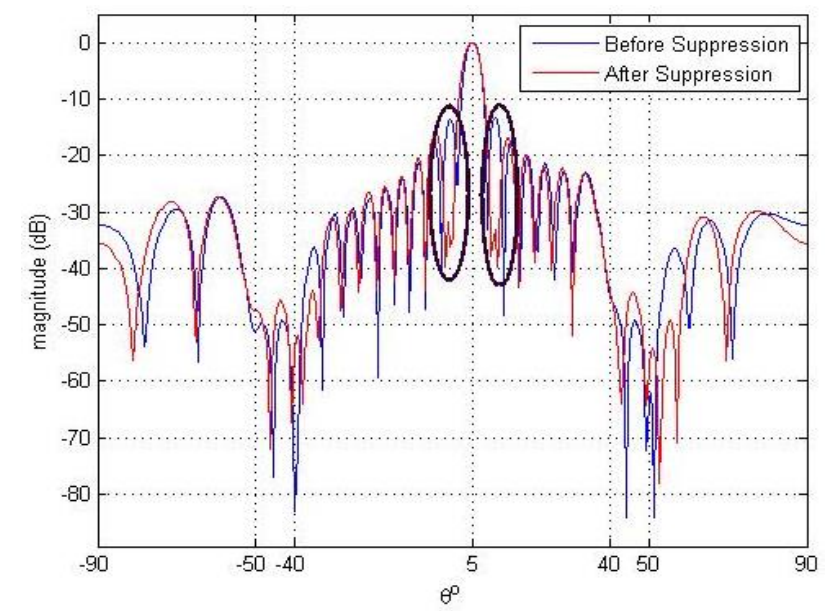

Fig. 6: Beampatterns before and after suppressing the sidelobe levels

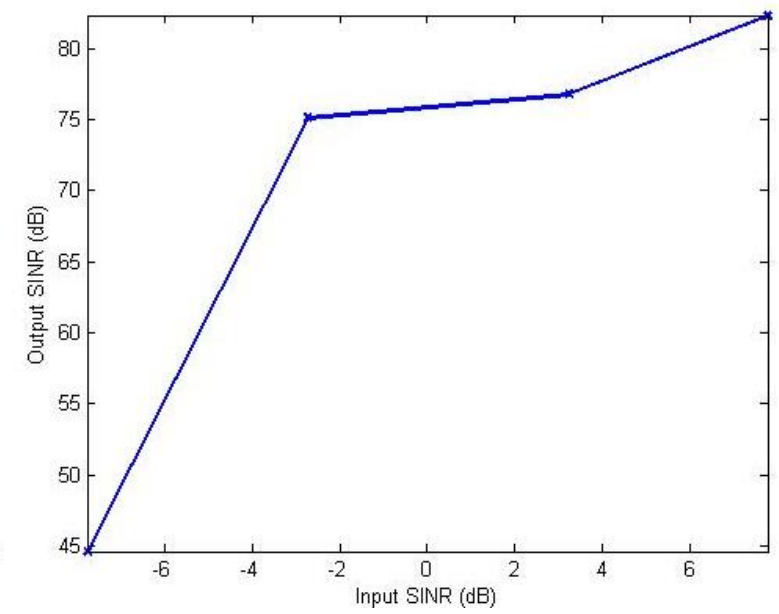

Fig. 7: Input SINR versus output SINR

\section{Conclusions}

The projected iterative MVDR (PI-MVDR) beamformer is proposed to enhance the conventional MVDR beamformer. The improvement can overcome the inverse covariance matrix avoidance, support available low number of samples, broaden nulls and suppress the first sidelobe levels close to the mainlobe. The covariance matrix used in the PI-MVDR beamformer is an average of between the estimated interferenceplus-noise covariance matrix and the sample covariance matrix of the received signal. In the presence of DOA mismatch, low number of samples circumstance and high input SNR, the PI-MVDR is capable of achieving all purposes after a few iterations. In addition, the averaged covariance matrix gives the better results than only using the estimated interference-plus-noise covariance matrix.

\section{References}

[1] J. R. Guerci, "Theory and application of covariance matrix tapers for robust adaptive beamforming," IEEE Transactions on Signal Processing, vol. 47, no. 4, pp. 977-987, Apr. 1999.

[2] W. Li, Y. Zhou, Q. Ye and B. Yang (October 2017). Adaptive antenna null broadening beamforming against array calibration error based on adaptive variable diagonal loading. International Journal of Antennas and Propagation. pp. 1-9. Available: http://doi.org/10.1155/2017/3265236

[3] X. Mao, W. Li, Y. Li. Y. Sun and Z. Zhai (2015). Robust adaptive beamforming against signal steering vector mismatch and jammer motion. International Journal of Antennas and Propagation. pp. 1-12. Available: http://doi.org/10.1155/2015/780296

[4] I. P. Gravas, Z. D. Zaharias, T. V. Yioultsis, P.I. Lazaridis and T. D. Xenos, "Adaptive beamforming with sidelobe suppression by placing extra radiation pattern nulls," IEEE Transactions on Antennas and Propagation, vol. 67, no. 6, pp. 3853-3861, Jun. 2019.

[5] F. Liu, G. Sun, J. Wang and R. Du (April 2014). Null broadening and sidelobe control algorithm via multiparametric quadratic programming for robust adaptive beamforming. ACES Journal, 29(4). pp. 307-315. Available: https://aces-society.org/search.php?vol=29\&no=4\&type $=2$

[6] F. Liu, Y. Wu, H. Duan and R. Du (2018). SVR-CMT algorithm for null broadening and sidelobe control. Progress in Electromagnetics Research. 163. pp. 39-50. Available: http://www.jpier.org/PIER/pier.php?volume=163

[7] S. A. Vorobyov, "Principles of minimum variance robust adaptive beamforming design," Signal Processing, vol. 93, pp. 3264-3277, 2013.

[8] M. Emadi, K.H. Sadeghi, A. Jafargholi and F. Marvasti (2008). Cochannel interference cancellation by the use of iterative digital beam forming method. Progress in Electromagnetics Research. 87. pp. 89-103. Available: http://www.jpier.org/PIER/pier.php?volume=87

[9] B. Ors and R. Suleesathira, "First and second order iterative null broadening beamforming," $3{ }^{\text {rd }}$ International 
Conference on Imaging, Signal Processing and Communication, Singapore, Jul. 27-29, 2019.

[10] B. Ors and R. Suleesathira, "Iterative broad null steering," The $3^{\text {rd }}$ International Conference on Graphics and Signal Processing, Hong Kong, Jun. 1-3. 2019.

[11] Q. Luo, J. Xie, H. Li and Z. He, "Robust adaptive beamforming in the presence of strong desired signal and DOA mismatch,” International Conference on Computational Problem-solving, China, Oct. 26-28, 2013.

[12] K. Yang, Z. Zhao and Q. H. Liu (2013). Robust adaptive beamforming against array calibration errors. Progress in Electromagnetics Research. 140. pp. 341-351. Available: http://www.jpier.org/PIER/pier.php?volume=140

[13] Y. Hou, L. Xue and Y. Jin, "Robust adaptive beamforming method based on interference-plus-noise covariance matrix," International Conference on Signal Processing, Communications and Computing, China, Aug. 5-8, 2013. 